UVX 2010 (2011) 115-121

DOI: $10.1051 / \mathrm{uvx} / 2011016$

(C) Owned by the authors, published by EDP Sciences, 2011

\title{
X-UV spectroscopy for nuclear magnetic fusion diagnostic
}

\author{
O. Meyer, R. Guirlet, S. Haquin and P. Monier-Garbet \\ CEA/Institut de Recherche sur la Fusion Magnétique, 13108 Saint paul Lez Durance Cedex, \\ France
}

\begin{abstract}
Magnetic fusion research is on the way to face a major challenge with the realization of the International Thermonuclear Experimental Reactor (ITER), which aims to demonstrate its technological feasibility. Given the range of temperatures encountered in fusion plasmas, X-UV spectroscopy has become a mandatory diagnostic tool for such plasma experiments. Various parameters such as impurity densities, ion velocities, electrons and ions temperatures are inferred through analysis of specific spectral lines or continuum levels. The ITER project imposes however new constraints due mainly to the nuclear environment and requires also new technological developments.
\end{abstract}

\section{MAGNETIC CONFINMENT NUCLEAR FUSION RESEARCH}

The goal of nuclear fusion research is to perform fusion of hydrogen isotopes to provide a sustainable energy source for the future generations. The most efficient reaction concerns Deuterium and Tritium hydrogen isotopes which react as follows:

$$
\mathrm{T}+\mathrm{D} \rightarrow{ }^{4} \mathrm{He}(3.56 \mathrm{MeV})+\mathrm{n}(14.03 \mathrm{MeV})
$$

Two criteria govern the reaction effectiveness: the temperature should be above $1 \mathrm{keV}$ deduced from cross-section calculations shown on figure 1 and the Lawson criterion that concerns both electronic density $\left(\mathrm{n}_{\mathrm{e}}\right)$ and energy confinement time $\left(\mathrm{W}_{\mathrm{th}} / \mathrm{P}_{\text {losses }}\right)$ where $\mathrm{W}_{\text {th }}$ is the energy stored in the plasma and $\mathrm{P}_{\text {losses }}$ is the power of the losses:

$$
\left(\mathrm{W}_{\text {th. plasma }} / \mathrm{P}_{\text {losses }}\right) \cdot \mathrm{n}_{\mathrm{e}}>6.10^{20} \mathrm{~s} \cdot \mathrm{m}^{-3}
$$

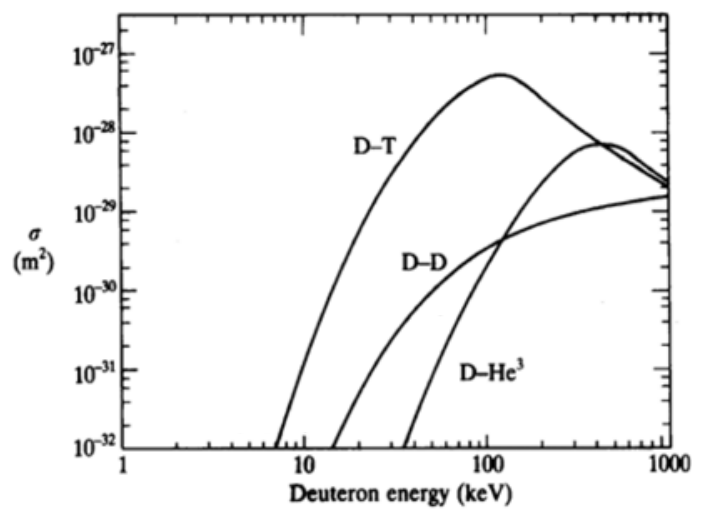

Figure 1. Nuclear fusion reaction cross sections.

This is an Open Access article distributed under the terms of the Creative Commons Attribution-Noncommercial License 3.0, which permits unrestricted use, distribution, and reproduction in any noncommercial medium, provided the original work is properly cited. 
Research addresses two different ways to provide fusion environment conditions: the "inertial confinement" branch where the conditions are obtained using electromagnetic confinement induced by lasers and the magnetic confinement branch, which is the object of this article.

\subsection{Magnetic confinement principle}

To realize fusion reactions by magnetic confinement the temperature criterion imposes to work in a plasma environment. Various magnetic confinement configurations have been developed; the most widely used being the Tokamak device that sets up a magnetic confinement by the superposition of two magnetic field components as shown in figure 2. Regarding the plasma shaping, the magnetic configuration exploited on most of the present Tokamak devices is the divertor configuration. That configuration, described in figure 3, permits to separate the plasma surface interaction area (denoted 3 in the figure), and the fusion reaction location area (denoted 1 in the figure). The plasma is actually divided into three main regions: the central region is the location of the fusion reaction; it is characterized by closed magnetic surfaces providing confinement times from 0.1 to 5 seconds. The typical electronic temperature range is $100 \mathrm{eV}$ to $10 \mathrm{keV}$. Low $\mathrm{Z}$ impurities are totally ionised and high $\mathrm{Z}$ impurities radiate in the UV-X range. The edge area (denoted 2 in the figure) exhibits open magnetic surfaces with typical electronic temperatures between 1 to $100 \mathrm{eV}$. Here, both low and high $\mathrm{Z}$ impurities radiate in the visibleUV range. The last area is the divertor region which is the main plasma-surface interaction location. The particles are neutralized on the divertor targets and then pumped out of the plasma. Power fluxes up to $10 \mathrm{MW} / \mathrm{m}^{2}$ can be achieved and suitable plasma-facing components are then mandatory. The divertor region constitutes the main plasma impurity source and electron temperatures below $10 \mathrm{eV}$ generate mostly visible radiations.

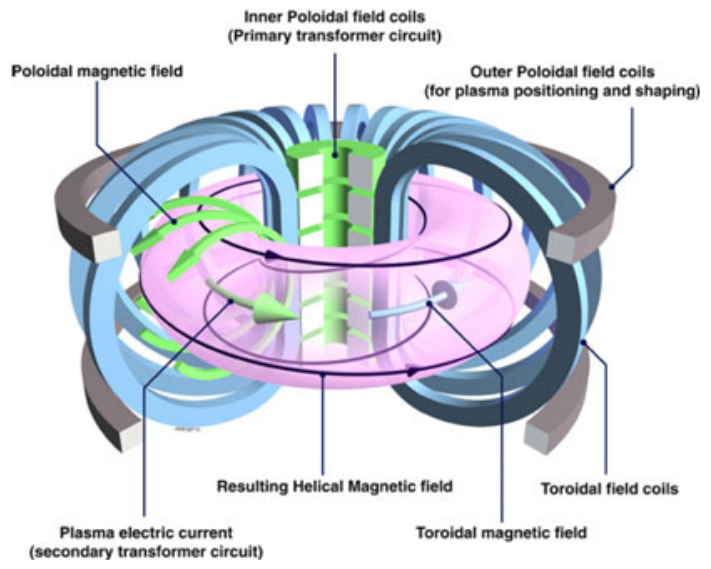

Figure 2. Tokamak magnetic confinement principle [7].

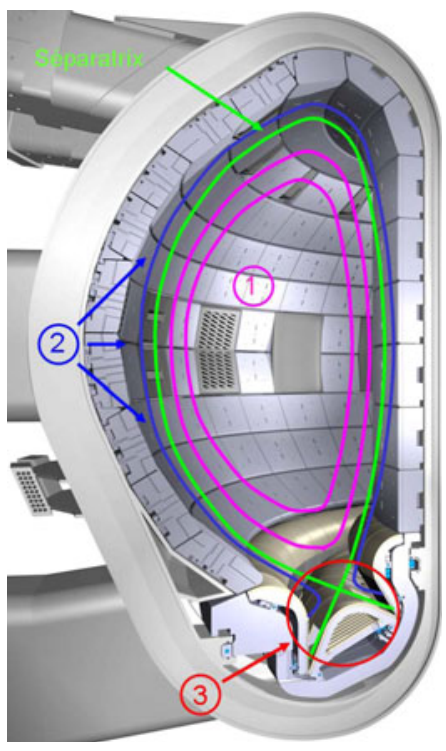

Figure 3. The divertor magnetic configuration.

\subsection{Impurity issues in magnetic fusion plasmas}

The impurities present in the plasma constitute a major issue for fusion plasma operation. In the centre of the plasma, they induce a fuel dilution and radiation losses for medium and high $\mathrm{Z}$ impurities. In the plasma edge, impurity radiation losses present the advantage to spread the power deposited on plasma 
facing components and thus avoid damaging localized power fluxes. The plasma facing components constitute the major impurity source and their choice has always been a critical issue for tokamaks design. The two main parameters in balance when choosing a material are radiating capability and the electron temperature erosion threshold. The figure 4 shows the radiative loss function for different elements in a plasma environment. The radiative losses roughly increase as $\mathrm{Z}^{3}$ which would make preferable the use of low $\mathrm{Z}$ materials. However, such materials are characterized by lower temperature erosion threshold than high $\mathrm{Z}$ materials.

The future International Thermonuclear Experimental Reactor (ITER) will include a Beryllium wall and Tungsten (W) divertor targets. In the $\mathrm{W}$ divertor an electron temperature below $10 \mathrm{eV}$ will be required to avoid $\mathrm{W}$ erosion and induced plasma radiative losses.

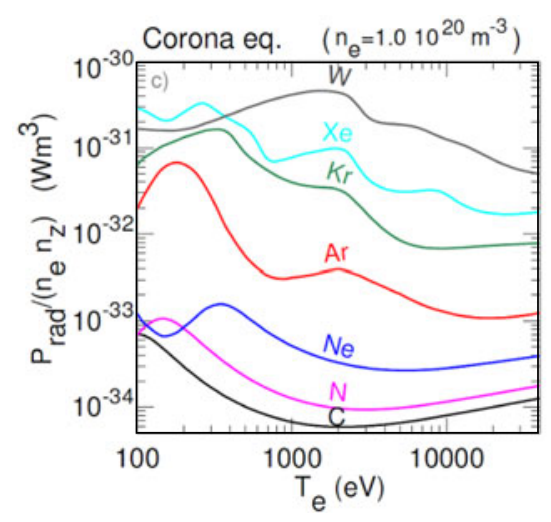

Figure 4. Coronal equilibrium radiative loss function (core conditions) [3].

\section{X-UV PLASMA SPECTROSCOPIC MEASUREMENTS TECHNIQUES}

Given the temperature range observed in fusion plasmas, the X-UV spectroscopy constitutes a major diagnostic tool. Both lines and continuum spectrum are exploited to determine different plasma parameters. X-UV electron impact excited lines spectra are used to derive impurity densities, Maxwellian electron and ion temperatures as well as plasma rotation velocities. The spectra are obtained using spectrometers with diffracting elements. The crucial parameter for such diagnostic techniques is generally the resolving power $\lambda / \Delta \lambda$ that affects line shape analysis and line discrimination. For the UV range, spectrometers using focalized gratings in grazing incidence are generally used; concerning X-ray range, Bragg crystal spectrometers have been chosen.

The $\mathrm{X}$ continuum spectrum, due essentially to Bremsstrahlung and radiative recombination emissions, is used to diagnose global impurity contamination in the centre of the plasma and to study electron populations. For X-ray continuum measurements, counting techniques on energy bands are used. The high efficiencies of the detection systems permit to obtain submillisecond acquisition times and to realize tomographic reconstructions by the use of numerous lines of sight.

\subsection{Plasma parameters deduced from lines spectrum}

\subsubsection{Impurity densities}

The first parameter to take into account when analysing plasma impurities is the ionization equilibrium: impurity neutrals entering the plasma undergo successive ionizations linked to the different electron temperatures encountered. Ionisation equilibrium calculations as shown on figure 5 for oxygen must 
be used for the impurity content analysis. Regarding instrumental aspects, spectrometers generally use integrated lines of sight and see numerous ionisation state emissions. Sufficient resolving power is thus necessary to separate the lines $(\lambda / \Delta \lambda \sim 1500$ for UV spectrometers).

Impurity densities are estimated using the line emissivity of impurity ions. The local density of an ion of charge $\mathrm{Zn}_{\mathrm{z}}(\mathrm{r}, \mathrm{t})$ is linked to the local emissivity $\mathrm{E}_{\mathrm{z}}^{\mathrm{n}}(\mathrm{r}, \mathrm{t})$ of the line $\mathrm{n}$ of that ion by the equation:

$$
E_{z}^{n}(r, t)=P E C_{n}\left(n_{e}, T_{e}\right) n_{e}(r, t) n_{z}(r, t)
$$

Where $\mathrm{n}_{\mathrm{e}}$ and $\mathrm{T}_{\mathrm{e}}$ are the local electron temperature and density; $\mathrm{PEC}_{\mathrm{n}}$ is the Photo Emission Coefficient of the line $\mathrm{n}$ that is provided by atomic data calculations like those of the ADAS database [5].

One issue is also to deduce the local emissivity from the integrated one; that can be done by using inversion techniques like Abel inversion that need several lines of sight. Other issues can be to get relevant local measurements of $\mathrm{n}_{\mathrm{e}}$ and $\mathrm{T}_{\mathrm{e}}$ and atomic data.

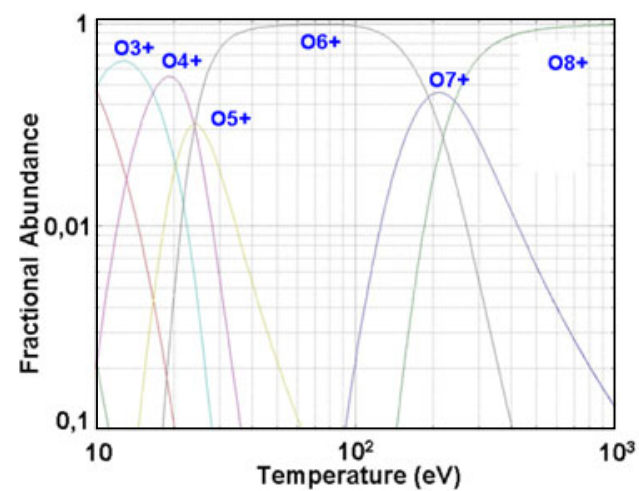

Figure 5. Oxygen ionization state distribution from a Coronal equilibrium calculation.

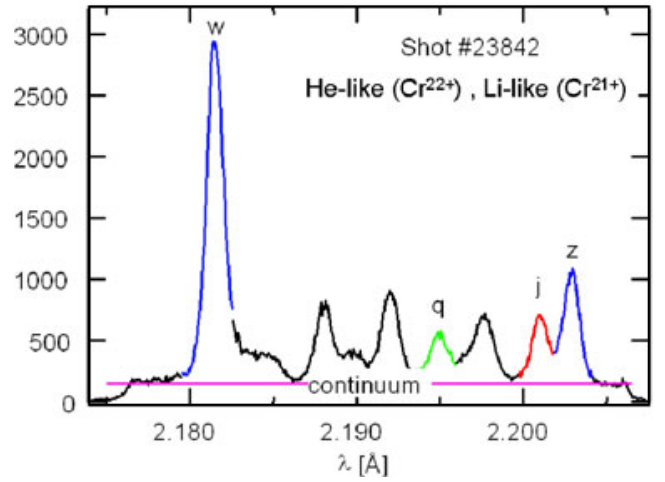

Figure 6. Spectrum obtained with the Tore Supra Bragg spectrometer.

\subsubsection{Central ion temperature and plasma rotation velocities}

Central ion temperature and plasma rotation can be obtained using the $\mathrm{X}$ spectrum measured by Bragg spectrometers as shown on figure 6 where rotation velocity and thermal ion temperature are deduced from Doppler shift and broadening respectively. Such measurements benefit from the high resolving powers $(\lambda / \Delta \lambda \sim 10000)$ that can be obtained by Bragg spectrometers.

\subsubsection{Central electron temperature}

Electron temperature can also be deduced from Bragg spectra as shown in figure 6 using line ratio of the " $\mathrm{j}$ " Li-like satellite line and the resonance He-like line "w" [4]. The knowledge of the ionisation equilibrium is crucial in such applications because the integrating line of sight measurement does not provide the location of the emission.

\subsection{Plasma parameters deduced from continuum}

As mentioned above, the continuum radiation is due to bremsstrahlung and radiative recombination and also strongly depends on electron populations. Plasma continuum radiation generally presents two main components: the thermal component due to the thermal electronic population that concern roughly the soft X-ray range (4-20 keV) and the suprathermal component (above $20 \mathrm{keV}$ ). 


\subsubsection{Thermal range}

Soft X-ray measurement consists of wide spectral range integration ( $\sim 5$ to $20 \mathrm{keV})$ and is dependant on several plasma parameters. The total power emitted by bremsstrahlung due to the thermal electron population can also be expressed as:

$$
P_{\text {brem }} \propto Z^{2} \cdot n_{e} \cdot n_{z} \cdot \sqrt{\mathrm{T}_{\mathrm{e}}}
$$

Data analysis must be carried out with care. Soft X-ray measurements are used for central impurity density evaluation: it is the only way to measure the low $\mathrm{Z}$ impurities that are fully stripped in the plasma centre. Concerning high $\mathrm{Z}$ impurities, a line spectrum contribution has to be taken into account if the considered ions are not fully stripped.

Another application of soft X-ray measurements is Magneto Hydro Dynamic (MHD) characterisation. The MHD activity modifies the magnetic topology of the plasma and induces a strong signature on the soft X-ray measurements as shown in figure 7. Such measurements are also useful to detect instabilities that can induce violent current breakdowns; prospection is in progress in order to do induce smooth current shutdowns when such instabilities are detected by soft X-ray diagnostics.

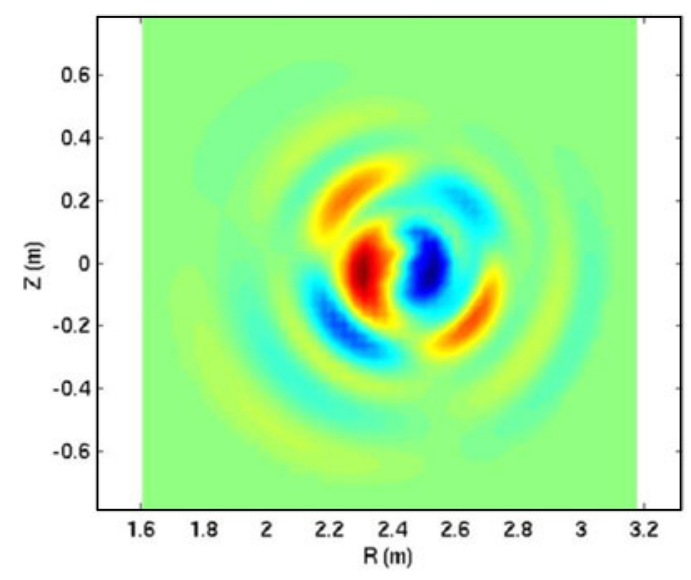

Figure 7. Magnetic islands detected by the Tore Supra soft X-ray system.

\subsubsection{Supra thermal range}

Hard X-ray diagnostic measures X-ray emission from $20 \mathrm{keV}$ to $200 \mathrm{keV}$ with an energy resolution of about $10 \mathrm{keV}$. A first application of the system is the detection of X-ray emission generated by high energy electrons called "runaway". Because of their huge energies (several MeV) those electrons can induce strong damages when interacting with the inner wall components. The early detection of these high energy electrons permits to do a soft plasma shutdown to avoid inner wall damages.

The X-ray diagnostic is also used to optimize non-inductive plasma scenarii. In these scenarii, the current is generated by producing a fast electron population through dedicated wave coupling. The plasma current increase results from the fast electrons population production. The X-ray diagnostic with its tomography feature is an asset to optimize the wave deposition location by tracking the associated fast electron population signature.

\section{THE ITER PROJECT}

The ITER project is a partnership of seven countries (European Union, China, India, Japan, Korea, Russia and United States of America) and its goal is to pass another milestone in the magnetic fusion 
feasibility demonstration by showing that plasma fusion conditions can be maintained by the ${ }^{4} \mathrm{He}$ particles deposited energy. A total fusion power of $500 \mathrm{MW}$ will have to be obtained with an efficiency $\mathrm{P}_{\text {fus }} / \mathrm{P}_{\mathrm{inj}}=10$. For comparison, the Joint European Torus (JET), the most powerful of present Tokamaks, has produced $26 \mathrm{MW}$ of fusion power with an efficiency ratio of 1 .

\subsection{Constraints linked to the project}

The ITER project is much more constraining than the present facilities for two main reasons. The first one is linked to the challenging performances expected in terms of fusion power: ITER will have to operate close to its operating margins and that requests real time control of plasma parameters with fast and reliable diagnostic systems. The second reason concerns the nuclear environment: the future ITER tokamak will be concerned with high energy neutron irradiance of $3.10^{8} \mathrm{~m}^{-2} \mathrm{~s}^{-1}$ inducing new issues for diagnostic development like cables radiation induce conductivity and EMF or radiation induced absorption and luminescence in optical materials [1]. Specific research and development are also required in those areas. Another issue linked to neutron irradiance is the structures activation that will replace the classic systems handling by remote operations. More generally, the systems will have to be deported behind protecting shields and input mirrors.

Table 1. Characteristics of X-UV diagnostics planned for ITER [2].

\begin{tabular}{|l|l|l|l|}
\hline Instrument & $\begin{array}{l}\text { Wavelength/ } \\
\text { Energy Range }\end{array}$ & $\begin{array}{l}\text { Regions Probed/ } \\
\text { Viewing Directions }\end{array}$ & Function \\
\hline VUV (main plasma) & $2.3-160 \mathrm{~nm}$ & $\begin{array}{l}\text { Upper and equatorial } \\
\text { regions; divertor region }\end{array}$ & $\begin{array}{l}\text { Impurity species } \\
\text { identification (core) and } \\
\text { impurity profile (Edge) }\end{array}$ \\
\hline $\begin{array}{l}\text { X-ray spectroscopy } \\
\text { (high resolution) }\end{array}$ & $0.05-0.5 \mathrm{~nm}$ & Core and edge & $\begin{array}{l}\text { Impurity species } \\
\text { identification, plasma } \\
\text { rotation, } \mathrm{T}_{\mathrm{i}}\end{array}$ \\
\hline Radial X-Ray Camera & $1-200 \mathrm{keV}$ & Full poloidal profile & $\begin{array}{l}\text { MHD, Impurity influxes, } \\
\text { Te influxes, Te }\end{array}$ \\
\hline $\begin{array}{l}\text { a- X-Ray Spectrometry } \\
\text { (Survey) } \\
\text { b- X-ray spectroscopy } \\
\text { (high resolution) }\end{array}$ & a) $0.1-10 \mathrm{~nm}$ \\
b) $0.1-1.0 \mathrm{~nm}$ & $\begin{array}{l}\text { a) Central chord } \\
\text { b) Edge }\end{array}$ & $\begin{array}{l}\text { Impurity species } \\
\text { identification, plasma } \\
\text { rotation, } \mathrm{T}_{\mathrm{i}} \text {. }\end{array}$ \\
\hline $\begin{array}{l}\text { Hard X-ray Monitor } \\
\text { (H-Phase) }\end{array}$ & $100 \mathrm{keV-20} \mathrm{MeV}$ & $\begin{array}{l}\text { One or more locations } \\
\text { on equatorial plane }\end{array}$ & $\begin{array}{l}\text { Runaway electron } \\
\text { detection }\end{array}$ \\
\hline $\begin{array}{l}\text { Divertor Spectroscopy } \\
\text { (VUV) }\end{array}$ & $15-40 \mathrm{~nm}$ & Divertor outer leg & $\begin{array}{l}\text { Divertor impurity } \\
\text { influxes, particularly } \\
\text { Tungsten }\end{array}$ \\
\hline
\end{tabular}

The operation with Tritium is another difference with present devices. ITER operation will be limited during its whole life to a strict Tritium inventory for the vacuum chamber components. This Tritium inventory will be another issue for systems directly connected to the vacuum vessel. The concept finally retained to take into account both constraints is the realization of removable port plugs where the diagnostic systems are gathered. The whole port plug can be disconnected from the vacuum chamber and brought in a dedicated cell where remote interventions can be done.

\subsection{X-UV Diagnostics On ITER}

X-UV diagnostics on ITER will be based on the ones already in use on present devices where diagnostic physics techniques are already well known. Alternative technical solutions have however to be found to match specific ITER requirements. Table 1 describes the measurements and analyse techniques 
planned for the ITER X-UV diagnostic systems. All of those diagnostics use measurements and analyse techniques described above.

As an example, let us focus on the "VUV main plasma Impurity profile (edge)" spectrometer shown in figure 8 that will be in charge of the edge impurity profiles measurements. The chosen spectral range of 21 to $26 \mathrm{~nm}$ is relevant to measure main expected ITER plasma impurities. Other specifications are a resolving power of 1500 , a temporal resolution of $10 \mathrm{~ms}$ and a spatial resolution of $50 \mathrm{~mm}$ for a radial range of $116 \mathrm{~cm}$. The technical solution is based on a toroidal grating assembly with variable line spacing providing flat image plan and corrected aberrations described on figure 9 [6]. Light is collected by a field cylindrical mirror and then imaged on the entrance slit using a toroidal mirror. After dispersion by the grating, the light is collected on a detection system. The detection system is still to be defined. The options are Micro-Channel Plates (MCP) coupled to a CCD camera or back-illuminated CCD. The main advantage of the MCP regarding back-illuminated CCD is the efficiency but its drawbacks are restrictive operation regarding vacuum aspects, relatively short life time and the need for appropriate high voltage supply.

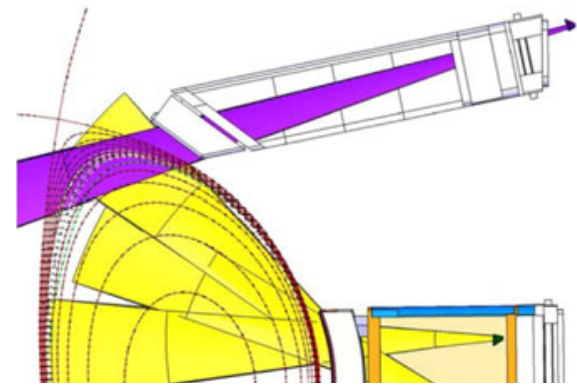

Figure 8. Location of the VUV impurity edge profiles diagnostic [2].

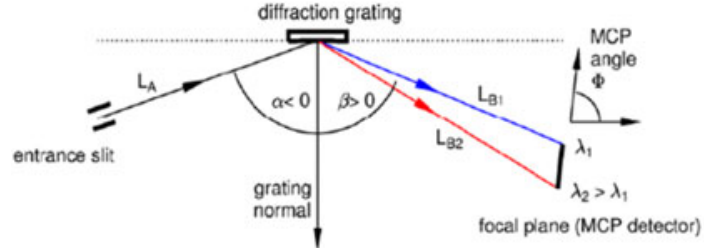

Figure 9. VUV flat field grating assembly [6].

\section{CONCLUSION}

$\mathrm{X}$-UV spectroscopy is an essential tool to determine important magnetic fusion plasma parameters such as impurity density, ion temperature, plasma rotation velocities, electron population or electron temperature. The future ITER facility requires diagnostic techniques, which are already well known and tested on present facilities but should also be operational in a much more restrictive environment (reduced operational margins, nuclear constraints ...). Technological developments will also be required to increase the long term reliability of the systems, to reduce their radiation sensitivity and to improve the mirrors technology.

\section{References}

[1] ITER Physics Basis, Nucl. Fusion 47 (2007) S1, S18, S337

[2] R. Reichle et al., Plant Description, Chap. 8: Diagnostics, ITER Organisation, 2009

[3] A. Kallenbach et al., Plasma Surface Interaction conference, San Diego, USA, 2010

[4] C. de Michelis, M. Mattioli, Nuclear Fusion 6 (1981)

[5] http://www.adas.ac.uk

[6] W. Biel, Rev. Sci. Instrum., 75, 3268 (2004)

[7] http://users.jet.efda.org 